\title{
A tutorial on Quantum Cohomology
}

\author{
Alexander Givental
}

\section{UC Berkeley}

Let $(M, f, G)$ be a manifold, a function and a Riemann metric on the manifold. Topologists would use these data in order to analyze the manifold by means of Morse theory, that is by studying the dynamical system $\dot{x}=$ $\pm \nabla f$. Many recent applications of physics to topology are based on another point of view suggested in E. Witten's paper Supersymmetry and Morse theory J. Diff. Geom. (1982).

Given the data $(M, f, G)$, physicists introduce some super-lagrangian whose bosonic part reads

$$
S\{x\}=\frac{1}{2} \int_{-\infty}^{\infty}\left(\|\dot{x}\|^{2}+\left\|\nabla_{x} f\right\|^{2}\right) d t
$$

and try to make sense of the Feynman path integral

$$
\int e^{i S\{x\} / \hbar} \mathcal{D}\{x\}
$$

Quasi-classical approximation to the path integral reduces the problem to studying the functional $S$ near its critical points, that is solutions to the 2-nd order Euler-Lagrange equations schematically written as

$$
\text { (1) } \ddot{x}=f^{\prime \prime} \nabla f \text {. }
$$

However a fixed point localization theorem in super-geometry allows further reduction of the problem to a neighborhood of those critical points which are fixed points of some super-symmetry built into the formalism. The invariant critical points turn out to be solutions of the 1-st order equation

$$
\text { (2) } \dot{x}= \pm \nabla f
$$


studied in the Morse theory.

Two examples:

- Let $M$ be the space of connections on a vector bundle over a compact 3-dimensional manifold $X$ and $f=C S$ be the Chern-Simons functional. Then (1) is the Yang-Mills equation on the 4-manifold $X \times \mathbb{R}$, and (2) is the (anti-)selfduality equation. Solutions of the anti-selfduality equation (called instantons) on $X \times \mathbb{R}$ are involved into the construction of Floer homology theory in the context of low-dimensional topology.

- Let $M$ be the loop space $L X$ of a compact symplectic manifold $X$ and $f$ be the action functional. Then (1) is the equation of harmonic maps $S^{1} \times \mathbb{R} \rightarrow X$ (with respect to an almost Kähler metric) and (2) is the Cauchy-Riemann equation. Solutions to the Cauchy-Riemann equation (that is holomorphic cylinders in $X$ ) participate in the construction of Floer homology in the context of symplectic topology.

In both examples the points in $M$ are actually fields, and both Yang-Mills and Cauchy-Riemann equations admit attractive generalizations to spacetimes (of dimensions $3+1$ and $1+1$ respectively) more sophisticated then the cylinders. It is useful however to have in mind that the corresponding field theory has a Morse theory somewhere in the background.

In the lectures we will be concerned about the second example. Let us mention here a few milestones of symplectic topology.

- In 1965 V. Arnold conjectured that a hamiltonian transformation of a compact symplectic manifold $X$ has fixed points - as many as critical points of some function on $X$.

- In 1983 C. Conley \& E. Zehnder confirmed the conjecture for symplectic tori $\mathbb{R}^{2 n} / \mathbb{Z}^{2 n}$. In fact they noticed that fixed points of a hamiltonian transformation correspond to critical points of the action functional $\oint p d q-H(p, q, t) d t$ on the loop space $L X$ due to the Least Action Principle of hamiltonian mechanics, and thus reduced the problem to Morse theory for action functionals on loop spaces.

- In 1985 M. Gromov introduced the technique of Cauchy-Riemann equations into symplectic topology and suggested to construct invariants of symplectic manifolds as bordism invariants of spaces of pseudo-holomorphic curves.

- In 1987 A. Floer invented an adequate algebraic-topological tool for Morse theory of action functionals - Floer homology - and proved Arnold's conjecture for some class of symplectic manifolds. In fact there are two types of 
inequalities in Morse theory: the Morse inequality

$$
\text { \#(critical points }) \geq \operatorname{Betty} \operatorname{sum}(X)
$$

which uses additive homology theory and applies to functions with nondegenerate critical points, and the Lusternik-Shnirelman inequality

$$
\text { \# (critical levels })>\text { cup-length }(X)
$$

which applies to functions with isolates critical points of arbitrary complexity and requires a multiplicative structure.

- Such a multiplicative structure introduced by Floer in 1989 and called now the quantum cup-product can be understood as a convolution multiplication in Floer homology induced by composition of loops $L X \times L X \rightarrow L X$. It arises every time when a Lusternik-Shnirelman-type estimate for fixed points of hamiltonian transformations is proved. For instance, the 1984 paper by B. Fortune \& A. Weinstein implicitly computes the quantum cup-product for complex projective spaces, and the pioneer paper by Conley \& Zehnder also uses the quantum cup-product (which is virtually unnoticeable since for symplectic tori it coincides with the ordinary cup-product).

- The name "quantum cohomology" and the construction of the quantum cup-product in the spirit of enumerative algebraic geometry were suggested in 1989 by E. Witten and motivated by ideas of $1+1$-dimensional conformal field theory. Witten showed that various enumerative invariants proposed by Gromov in order to distinguish symplectic structures actually obey numerous universal identities - to regrets of symplectic topologists and benefits of algebraic geometers.

- Several remarkable applications of such identities to enumeration of holomorphic curves and especially the so called mirror conjecture inspired an algebraic - geometrical approach to Gromov - Witten invariants, namely Kontsevich's project (1994) of stable maps. The successful completion of the project in 1996 by several (groups of) authors (K. Behrend, B. Fantechi, J. Li \& G. Tian, Y. Ruan,...) and the proof of the Arnold-Morse inequality in general symplectic manifolds (K. Fukaya \& K. Ono, 1996) based on similar ideas make intersection theory in moduli spaces of stable maps the most efficient technique in symplectic topology.

Exercise. Let $z=p+i q$ be a complex variable and $z(t)=\sum_{k \in \mathbb{Z}} z_{k} \exp i k t$ be the Fourier series of a periodic function. Show that the symplectic area $\oint p d q$ is the indefinite 
quadratic form $\oint p d q=\pi \sum k\left|z_{k}\right|^{2}$ on the loop space $L \mathbb{C}$. Deduce that gluing Morse cell complexes from unstable disks of critical points in the case of action functionals on loop spaces $L X$ would give rise to contractible topological spaces. (This exercise shows that Morse-Floer theory has to deal with cycles of infinite dimension and codimension rather then with usual homotopy invariants of loop spaces.)

\section{Moduli spaces of stable maps}

Example: quantum cohomology of complex projective spaces. In quantum cohomology theory it is convenient to think of cup-product operation on cohomology in Poincare-dual terms of intersection of cycles. In these terms the fundamental cycle represents the unit element 1 in $H^{*}\left(\mathbb{C} P^{n}\right)$, a projective hyperplane represents the generator $p \in H^{2}\left(\mathbb{C} P^{n}\right)$, intersection of two hyperplanes represents the generator $p^{2} \in H^{4}\left(\mathbb{C} P^{n}\right)$, and so on. Finally, the intersection point of $n$ generic hyperplanes corresponds to $p^{n} \in H^{2 n}\left(\mathbb{C} P^{n}\right)$ and one more intersection with $p$ is empty so that $H^{*}\left(\mathbb{C} P^{n}\right)=\mathbb{Q}[p] /\left(p^{n+1}\right)^{1}$

Exercise. Check that the Poincare intersection pairing $\langle\cdot, \cdot\rangle$ is given by the formula

$$
\int_{\left[\mathbb{C} P^{n}\right]} \phi \wedge \psi=\frac{1}{2 \pi i} \oint \phi(p) \psi(p) \frac{d p}{p^{n+1}} .
$$

The structural constants $\langle a \cup b, c\rangle$ of cup-product count the number of intersections of the cycles $a, b, c$ in general position (taken with signs prescribed by orientations).

The structural constants $\langle a \circ b, c\rangle$ of the quantum cup-product count the number of holomorphic spheres $\mathbb{C} P^{1} \rightarrow \mathbb{C} P^{n}$ passing by the points $0,1, \infty$ through the generic cycles $a, b, c$. In our example they are given by the formulas

$$
\left\langle p^{k} \cup p^{l}, p^{m}\right\rangle=\left\{\begin{array}{ccl}
q^{0} & \text { if } & k+l+m=n \\
q^{1} & \text { if } & k+l+m=2 n+1 \\
0 & \text { otherwise } &
\end{array} .\right.
$$

The first row corresponds to degree 0 holomorphic spheres which are simply points in the intersection of the three cycles. The second row corresponds

\footnotetext{
${ }^{1}$ We will always assume that coefficient ring is $\mathbb{Q}$ unless another choice is specified explicitly.
} 
to straight lines: all lines connecting projective subspaces $p^{k}$ and $p^{m}$ form a projective subspace of dimension $n-k+n-m+1=l$ which meets the subspace $p^{l}$ of codimension $l$ at one point. The degree 1 of straight lines in $\mathbb{C} P^{n}$ is indicated by the exponent in $q^{1}$. In general the monomial $q^{d}$ stands for contributions of degree $d$ spheres.

Exercise. Check that higher degree spheres do not contribute to the structural constants $\left\langle p^{k} \circ p^{l}, p^{m}\right\rangle$ for dimensional reasons. Verify that the above structural constants indeed define an associative commutative multiplication $\circ$ on $H^{*}\left(\mathbb{C} P^{n}\right)$ and that the generator $p$ of the quantum cohomology algebra of $Q H^{*}\left(\mathbb{C} P^{n}\right)$ satisfies the relation $p^{n+1}=q$. Show that the evaluation of cohomology classes from $\mathbb{Q}[p, q] /\left(p^{n+1}-q\right)$ on the fundamental cycle can be written in the residue form

$$
\int_{\left[\mathbb{C} P^{n}\right]} \phi(p, q)=\frac{1}{2 \pi i} \oint \frac{\phi(p, q) d p}{p^{n+1}-q} .
$$

As we shell see, the relation $p^{n+1}=q$ expresses the following enumerative recursion relation:

the number of degree $d$ holomorphic spheres passing by given marked points $0,1, \ldots, n, n+1, \ldots, N$ through the given generic cycles $p, p, \ldots, p, a, \ldots, b$ equals the number of degree $d-1$ spheres passing by the points $n+1, \ldots, N$ through $a, \ldots, b$.

Thus the very existence of the quantum cohomology algebra has serious enumerative consequences.

A rigorous construction of quantum cohomology algebras is based on the concept of stable maps introduced by M. Kontsevich.

Stable maps. Let $(\Sigma, \epsilon)$ be a compact connected complex curve $\Sigma$ with at most double singular points and an ordered $k$-tuple $\epsilon=\left(\epsilon_{1}, \ldots, \epsilon_{k}\right)$ of distinct non-singular marked points. Two holomorphic maps $f:(\Sigma, \epsilon) \rightarrow X$ and $f^{\prime}:\left(\Sigma^{\prime}, \epsilon^{\prime}\right) \rightarrow X$ to an (almost) Kähler manifold $X$ are called equivalent if there exists an isomorphism $\phi:(\Sigma, \epsilon) \rightarrow\left(\Sigma^{\prime}, \epsilon^{\prime}\right)$ such that its composition with $f^{\prime}$ equals $f$. A holomorphic map $f:(\Sigma, \epsilon) \rightarrow X$ is called stable if it has no non-trivial infinitesimal automorphisms.

Examples. (a) The constant map of an elliptic curve with no marked points is unstable since translations on the curve are automorphisms of the map.

(b) The constant map of $\mathbb{C} P^{1}$ with $<3$ marked points is unstable since the group of fractional linear transformations of $\mathbb{C} P^{1}$ is 3-dimensional. Similarly, if $\Sigma$ has $\mathbb{C} P^{1}$ as an irreducible component carrying $<3$ special (= marked 
or singular) points, and the map $f$ is constant on this component, then $f$ is unstable.

Exercise. Prove that any other map is stable.

The arithmetical genus $g(\Sigma)$ is defined as the dimension of the cohomology space $H^{1}\left(\Sigma, \mathcal{O}_{\Sigma}\right)$ of the curve with coefficients in the sheaf of holomorphic functions. The genus 0 curves (called rational) are in fact bunches of $\mathbb{C} P^{1}$ 's connected by the double points in a tree-like manner.

Exercise. Express the arithmetical genus of $\Sigma$ via Euler characteristics of its irreducible components and the Euler characteristic of the graph whose vertices correspond to the components and edges - to the double points.

The degree $d$ of the map $f$ is defined as the total sum of the homology classes represented in $X$ by the fundamental cycles of the components. Thus the degree is an element in the lattice $H_{2}(X, \mathbb{Z})$. The example of the degree 2 rational curve in $\mathbb{C} P^{2}$ given by the affine equation $x y=$ const which degenerates to the union of two straight lines when const $=0$ illustrates the general rule: irreducible holomorphic curves can degenerate to reducible ones but in the limit the genus and degree are conserved.

The set of equivalence classes of stable maps to $X$ with fixed arithmetical genus $g$, fixed number $k$ of marked points and fixed degree $d$ can be provided with a natural structure of a compact topological space (due to Gromov's compactness theorem) and is called the moduli space of stable maps. We will denote $X_{k, d}$ the genus 0 moduli spaces (and will mostly avoid higher genus moduli spaces throughout the text).

Examples. (a) Let $X$ be a point. Then the moduli spaces are DeligneMumford compactifications $\overline{\mathcal{M}}_{g, k}$ of the moduli spaces of complex structures on the sphere with $g$ handles and $k$ marked points. The spaces $\overline{\mathcal{M}}_{1,0}$ and $\overline{\mathcal{M}}_{0, k}$ with $k<3$ are empty. $\overline{\mathcal{M}}_{0,3}$ is a point (why?). A generic point in $M_{0,4}$ represents the cross-ratio $\lambda$ of the ordered 4 -tuple $(0,1, \infty, \lambda)$ of distinct marked points in $\mathbb{C} P^{1}$. Of course, the Deligne-Mumford compactification restores the forbidden values $\lambda=0,1, \infty$ so that $\overline{\mathcal{M}}_{0,4} \simeq \mathbb{C} P^{1}$. These values however correspond to the 3 ways of splitting 4 marked points into two pairs to be positioned on the 2 components of $\Sigma=\mathbb{C} P^{1} \cup \mathbb{C} P^{1}$ intersecting at a double point.

(b) The moduli spaces $X_{n, 0}$ of constant maps are the products $X \times \overline{\mathcal{M}}_{0, k}$ (empty for $k<3$ ). 
(c) The grassmannian $\mathbb{C} G(2, n+1)$ of straight lines in $\mathbb{C} P^{n}$ is compact and thus coincides with $\mathbb{C} P_{0,1}^{n}$.

Exercises. (a) Identify $\overline{\mathcal{M}}_{0,5}$ with the blow-up of $\mathbb{C} P^{2}$ at 4 points.

(b) Show that the moduli space of rational maps to $\mathbb{C} P^{1} \times \mathbb{C} P^{1}$ of degree $d=(1,1)$ with no marked points is isomorphic to $\mathbb{C} P^{3}$. Is it the same as $\mathbb{C} P_{1,3}^{1}$ ?

(c) How many points in $\mathbb{C} P_{0,4}^{3}$ represent stable maps with the image consisting of 4 distinct straight lines passing through the same point?

Evaluation of a stable map $f:(\Sigma, \epsilon)$ at the marked points $\left(\epsilon_{1}, \ldots, \epsilon_{k}\right)$ defines the maps ev $=\left(\mathrm{ev}_{1}, \ldots, \mathrm{ev}_{k}\right): X_{k, d} \rightarrow X^{k}$. Forgetting the marked point $\epsilon_{i}$ gives rise to tautological maps $\mathrm{ft}_{i}: X_{k+1, d} \rightarrow X_{k, d}$ as well as forgetting the map $f$ corresponds to the map $X_{k, d} \rightarrow \overline{\mathcal{M}}_{0, k}$ called contraction. One should have in mind that forgetting $f$ or a marked point can break the stability condition. The actual construction of forgetting and contraction maps involves contracting of all the irreducible components of $\Sigma$ which has become unstable.

For example, consider the fiber of $\mathrm{ft}_{k+1}: X_{k+1, d} \rightarrow X_{k, d}$ over the point represented by $f:\left(\Sigma, \epsilon_{1}, \ldots, \epsilon_{k}\right) \rightarrow X$. A point in the fiber corresponds to a choice of one more marked point on $\Sigma$. Any choice will give rise to a stable map unless the point is singular or marked in $\Sigma$. However in the case of the choice $\epsilon_{k+1}=\epsilon_{i}$ one can modify $\Sigma$ by an extra component $\mathbb{C} P^{1}$ intersecting $\Sigma$ at this point, carrying both $\epsilon_{k+1}$ and $\epsilon_{i}$ and extend $f$ to this component as the constant map. Similarly, in the case of a singular choice one can disjoin the branches of $\Sigma$ intersecting at this point and connect them with an extracomponent $\mathbb{C} P^{1}$ carrying the marked point $\epsilon_{k+1}$. Both modifications give rise to stable maps. Now it is easy to see that the fiber of $\mathrm{ft}_{k+1}$ is isomorphic to $(\Sigma, \epsilon)$ (factorized by the finite group $A u t(f)$ of automorphisms of the map $f$ if they exist). In particular the map $\mathrm{ft}_{k+1}$ has $k$ canonical sections $\left(\epsilon_{1}, \ldots, \epsilon_{k}\right): X_{k, d} \rightarrow X_{k+1, d}$ defined by the marked points in $\Sigma$. Moreover, the evaluation map ev $\mathrm{ev}_{k+1}: X_{k+1, d} \rightarrow X$ restricted to the fiber defines on $(\Sigma, \epsilon)$ a map equivalent to $f$. Thus the diagram defined by the projection $\mathrm{ft}_{k+1}$, by the map ev $\mathrm{v}_{k+1}$ to $X$ and by the sections $\epsilon_{i}$ can be interpreted as the universal degree $d$ stable map to $X$ with $k$ universal marked points $\left(\epsilon_{1}, \ldots, \epsilon_{k}\right)$.

Suppose now that $X$ is a homogeneous Kähler space (such as projective spaces, grassmannians, ..., flag manifolds). Then (see M.Kontsevich (1994) and K. Behrend \& Yu. Manin (1996) ) the moduli space $X_{k, d}$ has a natural structure of a complex orbifold (= local quotients of manifolds by finite 
groups) of complex dimension

$$
\operatorname{dim} X_{k, d}=\operatorname{dim} X+\left(c_{1}\left(T_{X}\right), d\right)-3+k .
$$

Here $\left(c_{1}\left(T_{X}\right), d\right)$ denotes the value of the 1-st Chern class of the tangent bundle $T_{X}$ on the homology class $d$, and the formula follows from the RiemannRoch theorem on $\Sigma$ which allows to compute the dimension of the infinitesimal variation space of holomorphic maps $\mathbb{C} P^{1} \rightarrow X$.

The topology of orbifolds is similar to that of manifolds. In particular one can develop Poincare duality theory and intersection theory in $X_{k, d}$ using the fundamental cycle of the orbifold which is defined at least over $\mathbb{Q}$.

For general $X$ the moduli spaces can have singularities and components of different dimensions. Nevertheless one can define in the moduli space a rational homology class (called the virtual fundamental cycle, see for instance J. Li \& G. Tian (1996)) which has the Riemann-Roch dimension and allows to build intersection theory with the same nice properties as in the case of homogeneous Kähler spaces. The initial point in the definition of the virtual fundamental cycle is to understand that singularities of the moduli spaces mean irregularity of the zero value of the Cauchy-Riemann equation selecting holomorphic maps among all smooth maps. The cycle is to have the same properties as if the Cauchy-Riemann equations were made regular by bringing "everything" (including the almost complex structure) into general position.

Exercises. (a) Suppose that all fibers of a holomorphic vector bundle $V$ over a rational curve $\Sigma$ are spanned by global holomorphic sections. Prove that $H^{1}(\Sigma, V)=0$ and find the dimension of $H^{0}(\Sigma, V)$. Describe the tangent space at the point $[f]$ to (the Aut $(f)$-covering of the orbifold ) $X_{k, d}$ for homogeneous $X$.

(b) Consider the space $X$ of constant stable maps to $X$ of a given elliptic curve $E$ with one marked point as a subspace in the space of all smooth maps. Check that 0 is irregular value of the Cauchy-Riemann equation linearized along a constant map and show that the virtual fundamental class should have dimension 0 and be equal to the Euler characteristic of $X$.

\section{Gromov-Witten invariants}

Structural constants of the quantum cup-product on $H^{*}(X)$ are defined by

$$
\langle a \circ b, c\rangle:=\sum_{d} q_{1}^{d_{1}} \ldots q_{r}^{d_{r}} \int_{\left[X_{3, d}\right]} \operatorname{ev}_{1}^{*}(a) \wedge \operatorname{ev}_{2}^{*}(b) \wedge \operatorname{ev}_{3}^{*}(c)
$$


where $\left(d_{1}, \ldots, d_{r}\right)$ is the coordinate expression of the degree $d$ in a basis of the lattice $H_{2}\left(X, \mathbb{Z}\right.$ ) (which we assume isomorphic to $\mathbb{Z}^{r}$ ), the integral means evaluation of a cohomology class on the virtual fundamental cycle, and $a, b, c$ are arbitrary cohomology classes of $X$.

Exercises. (a) Show that symplectic area of a holomorphic curve in (almost) Kähler manifold is positive. Deduce that the semigroup $\mathrm{L} \subset H_{2}(X, \mathbb{Z})$ of degrees of compact holomorphic curves fits some integer simplicial cone in the lattice at least in the case of Kähler manifolds with $H^{2}(X)$ spanned by Kähler classes. (In fact the same is true for generic almost Kähler structures and therefore - for any almost Kähler $X$ if $\mathrm{\iota}$ means the semigroup spanned by those degrees which actually contribute to the structural constants.) Conclude from this that the structural constants are (at worst) formal power series in $q_{1}, \ldots, q_{r}$ with respect to an appropriate basis in the lattice $H_{2}(X)$.

(b) Make precise sense of the statement that $Q H^{*}(X)$ is a $q$-deformation of $H^{*}(X)$.

(c) Prove that the quantum cup-product o respects the following grading on $H^{*}(X, \mathbb{Q}[[q]])$ : cohomology classes of $X$ are assigned their usual degrees divided by 2 since we want to count dimensions of cycles in "complex" units, and the parameters $q_{i}$ are assigned the degrees in accordance with the rule $\operatorname{deg} q^{d}=\left(c_{1}\left(T_{X}\right), d\right)$.

One can define more general Gromov-Witten invariants

$$
\left\langle a_{1}, \ldots, a_{k}\right\rangle_{d}:=\int_{\left[X_{k, d}\right]} \operatorname{ev}_{1}^{*}\left(a_{1}\right) \wedge \ldots \wedge \mathrm{ev}_{k}^{*}\left(a_{k}\right)
$$

which have the meaning of

the number of degree $d$ holomorphic spheres in $X$ passing through generic cycles Poincare-dual to the classes $a_{1}, \ldots, a_{k}$.

Notice that the configuration of points mapped to the cycles is not specified, and thus the invariants differ from those which participate in our interpretation of the relation $p^{n+1}=q$ in $\mathbb{Q} H^{*}\left(\mathbb{C} P^{n}\right)$. In order to fix the configuration one should use the fundamental cycle $\left[\mathrm{ct}^{-1}(p t)\right]$ of a fiber of the contraction map ct : $X_{k, d} \rightarrow \overline{\mathcal{M}}_{0, k}$. More generally, let $A$ be a cohomology class in $\overline{\mathcal{M}}_{0, k}$. The GW-invariant

$$
A\left\langle a_{1}, \ldots, a_{k}\right\rangle_{d}:=\int_{\left[X_{k, d}\right]} \mathrm{ct}^{*}(A) \wedge \mathrm{ev}_{1}^{*}\left(a_{1}\right) \wedge \ldots \wedge \mathrm{ev}_{k}^{*}\left(a_{k}\right)
$$

has the enumerative meaning of

the number of pairs - a degree $d$ map $\mathbb{C} P^{1} \rightarrow X$, a configuration - such that the configuration belongs to a cycle Poincare-dual to $A$ and the map send 
it to the given cycles in $X$.

One can do even better. Consider the section $\epsilon_{i}: X_{k, d} \rightarrow X_{k+1, d}$ defined by the universal marked point. The conormal line bundle to the section pulled back to $X_{k, d}$ by the section itself will be called the universal cotangent line to the universal curve at the $i$-th marked point (why?). Thus we have $k$ tautological line bundles over $X_{k, d}$ and we denote $c^{(1)}, \ldots, c^{(k)}$ their 1-st Chern classes.

Let $T(c)=t_{0}+t_{1} c+t_{2} c^{2}+\ldots$ be a polynomial in one variable $c$ with coefficients $t_{i} \in H^{*}(X)$. Given $k$ such polynomials $T^{(1)}, \ldots, T^{(k)}$, we can introduce the GW-invariants (called gravitational descendents)

$$
A\left\langle T^{(1)}, \ldots, T^{(k)}\right\rangle_{d}:=\int_{\left[X_{k, d}\right]} \mathrm{ct}^{*}(A) \wedge \mathrm{ev}_{1}^{*} T^{(1)}\left(c^{(1)}\right) \wedge \ldots \wedge \mathrm{ev}_{k}^{*} T^{(k)}\left(c^{(k)}\right)
$$

whose enumerative meaning is not so obvious (see however Exercise (b) below).

Exercises. (a) Let $G$ be a compact Lie group. Equivariant cohomology $H_{G}^{*}(M)$ of a $G$-space $M$ is defined as the cohomology $H^{*}\left(M_{G}\right)$ of the homotopy quotient $M_{G}:=$ $(M \times E G) / G$ and is a module over the coefficient algebra $H_{G}^{*}(p t)=H^{*}(B G)$ of the $G$ equivariant theory. Suppose that points of the $G$-space $M$ have only finite stabilizers. Show that $H_{G}^{*}(M, \mathbb{Q})$ is canonically isomorphic to $H^{*}(M / G, \mathbb{Q})$. Use this fact in order to define the Chern classes $c^{(i)} \in H^{*}\left(X_{k, d}\right)$ over $\mathbb{Q}$ accurately, that is taking into account the automorphism groups $A u t(f)$ of stable maps.

(b) A holomorphic section of a line bundle $L$ over $X$ with the 1-st Chern class $p$ determines a section of the bundle $\mathrm{ev}_{k+1}^{*} L$ over the universal curve. Define the $l+1$ dimensional bundle over $X_{k, d}$ of $l$-jets of such sections at the 1-st universal marked point and compute the Euler class of this bundle in terms of $p$ and $c^{(1)}$. Interpret the number of degree $d$ spheres subject to tangency constraints of given orders with given generic hypersurfaces in $X$ in terms of gravitational descendents.

As it follows directly from the definition of the structural constants, the quantum cup-product is (super-)commutative ${ }^{2}$ and satisfies the following Frobenius property with respect to the intersection pairing:

$$
\langle a \circ b, c\rangle=\langle a, b \circ c\rangle \text {. }
$$

\footnotetext{
${ }^{2}$ We will understand commutativity and symmetricity in the sense of super-algebra and thus will further omit the prefix super. It is safe however to assume that cohomology of $X$ has trivial odd part for it is true in our examples of homogeneous Kähler spaces.
} 
Associativity the quantum cup-product can be then formulated as the symmetricity with respect to permutations of the indices $(1,2,3,4)$ in the following quadratic combination of the structural constants

$$
\sum_{\alpha, \beta}\left\langle a_{1} \circ a_{2}, \phi_{\alpha}\right\rangle \eta^{\alpha \beta}\left\langle\phi_{\beta}, a_{3} \circ a_{4}\right\rangle
$$

where $\sum \eta^{\alpha \beta} \phi_{\alpha} \otimes \phi_{\beta}$ is the coordinate expression for the Poincare-dual of the diagonal $\Delta \subset X \times X$ in terms of a basis $\left\{\phi_{\alpha}\right\}$ in $H^{*}(X)$ and the intersection matrix $\left(\eta^{\alpha \beta}\right)$ inverse to $\left(\eta_{\alpha \beta}\right):=\left(\left\langle\phi_{\alpha}, \phi_{\beta}\right\rangle\right)$.

The associativity property can be explained as follows. Consider the GWinvariant $A\left\langle a_{1}, a_{2}, a_{3}, a_{4}\right\rangle_{d}$ which counts the number of degree $d$ spheres with the configuration $(0,1, \infty, \lambda)$ of marked points mapped to the given 4 cycles. It is obviously symmetric in $(1,2,3,4)$ and does not depend on $\lambda$. Now let the cross-ratio $\lambda$ approach one of the exceptional values 0,1 or $\infty$. In the limit the same $\mathrm{GW}$-invariant receives another interpretation: it counts the number of pairs of maps $f^{\prime}, f^{\prime \prime}: \mathbb{C} P^{1} \rightarrow X$ of degrees $d^{\prime}+d^{\prime \prime}=d$ such that $f^{\prime}(\infty)=f^{\prime \prime}(\infty)$ and $f^{\prime}(0), f^{\prime}(1), f^{\prime \prime}(0), f^{\prime \prime}(1)$ belong to the given 4 cycles. Which pair of the cycles constrains $f^{\prime}$ and which - $f^{\prime \prime}$ depends however on the special value of the cross-ratio $\lambda$. Rewriting the diagonal constraint $f^{\prime}(\infty)=f^{\prime \prime}(\infty)$ in $X \times X$ in terms of $\phi_{\alpha} \otimes \phi_{\beta}$ and summing contributions of various degrees with the weights $q^{d}$ we arrive at the identity between the above quadratic expression of the structural constants and the GW-invariant

$$
\sum_{d} q^{d} A\left\langle a_{1}, a_{2}, a_{3}, a_{4}\right\rangle_{d}
$$

symmetric under permutations.

Exercise. (a) Formulate the above argument in terms the contraction map ct : $X_{4, d} \rightarrow \overline{\mathcal{M}}_{0,4}$ and the evaluation map ev ${ }_{3}^{\prime} \times \mathrm{ev}_{3}^{\prime \prime}: X_{3, d^{\prime}} \times X_{3, d^{\prime \prime}}$ and prove the associativity of the quantum cup-product for a homogeneous Kähler space.

(b) Apply the same argument to the contraction maps ct : $X_{k, d} \rightarrow \overline{\mathcal{M}}_{0, k}$ with $k>4$ in order to show that the $\mathrm{GW}$-invariant $\sum_{d} q^{d} A\left\langle a_{1}, \ldots, a_{k}\right\rangle_{d}$ counting the number of maps $\mathbb{C} P^{1} \rightarrow X$ sending $1, \ldots, k$ to $a_{1}, \ldots, a_{k}$ can be expressed in terms of multiple products in $Q H^{*}(X)$ as $\left\langle a_{1}, a_{2} \circ a_{3} \circ \ldots \circ a_{k}\right\rangle$.

(c) Considering the degeneracy of the complex structure on a genus $g$ Riemann surface $\Sigma$ to the curve of geometrical genus 0 with $g$ self-intersections, express the virtual number of holomorphic maps $\Sigma \rightarrow X$ in terms of the quantum cup-product.

The associativity identity and the above interpretation of multiple products in $Q H^{*}(X)$ are examples of universal relations between $G W$-invariants 
referred sometimes as composition laws or Witten-Dijkgraaf-Verlinde-Verlinde equations. Another universal identity reads:

$$
q_{i} \frac{\partial}{\partial q_{i}}\left\langle a, p_{j} \circ b\right\rangle=q_{j} \frac{\partial}{\partial q_{j}}\left\langle a, p_{i} \circ b\right\rangle
$$

where $\left(p_{1}, \ldots, p_{r}\right)$ in an integer basis in $H^{2}(X)$ dual to the basis in $H_{2}(X, \mathbb{Z})$ which we use for labeling the degrees $d$ by $\left(d_{1}, \ldots, d_{r}\right)$. Due to $q_{i} \partial q^{d} / \partial q_{i}=$ $d_{i} q^{d}$, it follows from the divisor equation

$$
\left\langle a, p_{i}, b\right\rangle_{d}=d_{i}\langle a, b\rangle_{d}
$$

which simply means that a degree $d$ sphere has intersection index $d_{i}$ with a codimension 2 cycle Poincare-dual to $p_{i}$.

Exercise. Prove the divisor equation by computing the push-forward $\left(\mathrm{ft}_{2}\right)_{*} \mathrm{ev}_{2}^{*}\left(p_{i}\right)$ from $X_{3, d}$ to $X_{2, d}$. Apply the same argument to 1 instead of $p_{i}$ in order to conclude that $10=\mathrm{id}$ and give the enumerative explanation of the latter statement.

The divisor equation has the following remarkable interpretation. Consider the system of 1-st order linear differential equations for a vector-function of $q$ with values in $H^{*}(X)$

$$
\text { (3) } \quad \hbar q_{i} \frac{\partial}{\partial q_{i}} \vec{s}=p_{i} \circ \vec{s}
$$

depending also on the parameter $\hbar$.

The system (3) is consistent for any non-zero value of the parameter $\hbar$.

Indeed, the differential equations mean that the vector-function $\vec{s}$ is annihilated by the connection operator

$$
\nabla_{\hbar}:=\hbar d-\sum_{i=1}^{r} p_{i} \circ \frac{d q_{i}}{q_{i}} \wedge .
$$

The operator consists of the De Rham differential $d$ and of the exterior multiplication by the matrix-valued differential 1-form $A^{1}:=\sum\left(p_{i} \circ\right) d \ln q_{i}$. The consistency condition means that the connection is flat for any $\hbar$ :

$$
\nabla_{\hbar}^{2}:=\hbar^{2} d^{2}-\hbar d A^{1}+A^{1} \wedge A^{1}=0 .
$$

This is equivalent to commutativity of the quantum multiplication operators $p_{i}$ ○ and to the element-wise closedness $d A^{1}=0$ of the matrix-values 1-form. The latter is guaranteed by the divisor equations. 
Consistency of a differential system means that solutions exist. The role of solutions of the system (3) in Gromov-Witten theory can be explained in terms of gravitational descendents. Consider the GW-invariants

$$
S_{\alpha \beta}:=\left\langle\phi_{\alpha}, e^{(p \ln q) / \hbar} \phi_{\beta}\right\rangle+\sum_{d \neq 0} q^{d}\left\langle\phi_{\alpha}, e^{(p \ln q) / \hbar} \frac{\phi_{\beta}}{\hbar-c}\right\rangle_{d} .
$$

Here $c$ is the 1-st Chern class of the universal cotangent line at the 2-nd (of the two) marked points so that $1 /(\hbar-c)=h^{-1}+c \hbar^{-2}+c^{2} \hbar^{-3}+\ldots$ is an example of the function $T(c)$ participating in the definition of gravitational descendents, and $p \ln q=p_{1} \ln q_{1}+\ldots+p_{r} \ln q_{r}$.

The matrix $\left(S_{\alpha \beta}\right)$ is a fundamental solution matrix of the linear differential system (3).

One of the ways to approach this statement begins with a closer look at the universal cotangent line at the last marked point over $X_{3, d}$. Since the sphere $\mathbb{C} P^{1}$ with 3 marked points has a canonical coordinate system, the universal cotangent line appears to be a trivial line bundle. Such a conclusion is false because of reducible curves, which means that the line bundle has a non-vanishing section over the part of $X_{3, d}$ corresponding to irreducible curves, and the class $c$ is represented by a divisor consisting of the compactifying components. However, if such a component corresponds to reducible curves $\Sigma=\mathbb{C} P^{1} \cup \mathbb{C} P^{1}$ with the 3 -rd marked point situated on the same $\mathbb{C} P^{1}$ as at least one of the others, then the trivializing argument still applies since this $\mathbb{C} P^{1}$ has 3 special points. We conclude that the divisor representing $c$ corresponds to the components where the first $\mathbb{C} P^{1}$ carries the 1-st and the 2-nd marked points, and the 3-rd marked point is located on the 2 -nd $\mathbb{C} P^{1}$. A more detailed analysis shows that the class $c$ in $X_{3, d}$ is represented by

$$
\sum_{d^{\prime}+d^{\prime \prime}=d}\left[X_{3, d^{\prime}}\right] \times_{\Delta}\left[X_{2, d^{\prime \prime}}\right]
$$

where $\Delta$ symbolizes the diagonal constraint $f^{\prime}(\infty)=f^{\prime \prime}(0)$. Similarly to the case of the associativity equation this factorization of the class $c$ implies that

$$
\sum_{d} q^{d}\left\langle a, p_{i}, T(c)\right\rangle_{d}=\sum_{\alpha \beta}\left\langle a, p_{i} \circ \phi_{\alpha}\right\rangle \eta^{\alpha \beta}\left(\left\langle\phi_{\beta}, T(0)\right\rangle+\sum_{d \neq 0} q^{d}\left\langle\phi_{\beta}, \frac{T(c)-T(0)}{c}\right\rangle_{d}\right) .
$$


The second step consists in relating $\left\langle a, p_{i}, T(c)\right\rangle_{d}$ with $\langle a, T(c)\rangle_{d}$ in a fashion similar to the divisor equation. At the first glance the invariants are related by the push-forward of $p_{i}$ along the map $\mathrm{ft}_{2}: X_{3, d} \rightarrow X_{2, d}$ and thus differ by the factor $d_{i}$. This conclusion is false because $\mathrm{ft}_{2}^{*}\left(c^{(3)}\right) \neq c^{(3)}$ on $X_{3, d}$. More precisely, the universal cotangent line $L$ at the last marked point coincides with the pull-back from $X_{2, d}$ of the universal cotangent line $L^{\prime}$ with the same name unless the last marked point coincides with the forgotten 2-nd marked point. Recalling the construction of forgetting maps we find that $L$ is trivial on the divisor $D:=\left[\epsilon_{3}\right]$ in $X_{3, d}$ while $\mathrm{ft}_{2}^{*} L^{\prime}$ restricted to $D \simeq X_{2, d}$ is equivalent to the universal cotangent line on $X_{2, d}$. This actually means that

$$
c_{1}\left(\mathrm{ft}_{2}^{*} L^{\prime}\right)=c_{1}(L)+[D] \text { and }[D] \cap c_{1}(L)=0 .
$$

We arrive at the following generalization of the divisor equation:

$$
\left\langle a, p_{i}, T(c)\right\rangle_{d}=d_{i}\langle a, T(c)\rangle_{d}+\left\langle a, p_{i} \frac{T(c)-T(0)}{c}\right\rangle_{d} .
$$

It is left only to notice that $T(c)=1 /(\hbar-c)$ is the eigen-function of the operation $(T(c)-T(0)) / c$ with the eigen-value $1 / \hbar$ and that the conclusion of the second step agrees with the differentiation of $S_{\alpha \beta}$ in $\ln q_{i}$.

Exercise. Give another, rigorous proof of the fundamental solution statement (in the case of homogeneous $X$ ):

(a) following the argument in the second step prove the string equation

$$
\langle a, 1, T(c)\rangle_{d}=\left\langle a, \frac{T(c)-T(0)}{c}\right\rangle_{d}
$$

(b) apply the 4-point argument to the descendent $A\left\langle a, p_{i}, 1, T(c)\right\rangle_{d}$ in order to prove that

$$
\sum_{\alpha \beta}\left\langle a, p_{i}, \phi_{\alpha}\right\rangle_{d}\left\langle\phi_{\beta}, 1, T(c)\right\rangle_{d}=\left\langle a, p_{i}, T(c)\right\rangle_{d}
$$

(c) deduce the differential equation for $\left(S_{\alpha \beta}\right)$ formally, using (a),(b) and the generalized divisor equation.

The differential equations for the gravitational descendents give one more example of universal identities between GW-invariants and along with some initial conditions (asymptotical at $q=0$ ) allows to recover the gravitational descendents from the structural constants of the quantum cohomology algebra. A more general theory involving other GW-invariants and gravitational descendents will be outlined in the exercises. 
Exercises. Define the genus 0 potential of $X$ as the following formal function of $q$ and $t \in H^{*}(X)$ :

$$
F(t, q)=\sum_{k=0}^{\infty} \frac{1}{k !} \sum_{d} q^{d}\langle t, t, \ldots, t\rangle_{d}
$$

Solutions to the following exercises can be obtained by slight modification of the arguments used in the proof of the WDVV, string and divisor equations.

(a) Express the GW-invariants $\left\langle a_{1}, \ldots, a_{k}\right\rangle_{d}$ as Taylor coefficients of $F$.

(b) On the space $H^{*}(X)$, define the quantum cup-product $o_{t}$ depending on $t$ by

$$
\left\langle a \circ_{t} b, c\right\rangle:=\partial_{a} \partial_{b} \partial_{c} F
$$

where $\partial_{v} F$ means the directional derivative of $F$ as a function of $t$ in the direction of the vector $v \in H^{*}(X)$. Prove that $\circ_{t}$ provides the cohomology space with the structure of commutative associative Frobenius algebra with unity 1 at least if $X$ is a homogeneous Kähler space. Find the degrees of the parameters $t, q$ which make the quantum cup-product graded.

(c) Show that the connection $\nabla_{\hbar}:=\hbar d-\sum_{\alpha}\left(\phi_{\alpha} \circ_{t}\right) \wedge$ (it can be understood as a connection on the tangent bundle of the manifold $\left.H^{*}(X)\right)$ is flat for any $\hbar \neq 0$.

(d) Introduce the potential

$$
S_{\alpha \beta}:=\sum_{k=0}^{\infty} \frac{1}{k !} \sum_{d} q^{d}\left\langle\phi_{\alpha}, t, \ldots, t, \frac{\phi_{\beta}}{\hbar-c^{(k+2)}}\right\rangle_{d}
$$

for the gravitational descendents. Prove that $\left(S_{\alpha \beta}\right)$ is a fundamental solution matrix for the differential system $\nabla_{\hbar} S=0$ (at least in the case of homogeneous Kähler spaces).

(e) Prove the following generalization of the divisor equation: for our basis $\left(p_{1}, \ldots, p_{r}\right)$ in $H^{2}(X)$ and any $a, b, c$

$$
\partial_{p_{i}} \partial_{a} \partial_{b} \partial_{c} F=q_{i} \frac{\partial}{\partial q_{i}} \partial_{a} \partial_{b} \partial_{c} F
$$

Analyze the relation between the $q$-deformation $\circ$ of the cup-product introduced at the beginning of the section 2 and the $t$-deformation $\circ_{t}$.

(f) Find generalizations of WDVV, string and divisor equations to gravitational descendents. Show that all the genus 0 descendents $\left\langle T^{1}, \ldots, T^{k}\right\rangle_{d}$ are determined by the potential $F$. Introduce the potential

$$
\mathcal{F}(T)=\sum_{k} \frac{1}{k !} \sum_{d} q^{d}\left\langle T\left(c^{(1)}\right), \ldots, T\left(c^{(k)}\right)\right\rangle_{d}
$$

for genus 0 gravitational descendents and try to describe the procedure expressing $\mathcal{F}$ in terms of $F$ (we refer to B. Dubrovin, The geometry of $2 D$ topological field theory for the answer).

(g) Introduce higher genus analogies $\mathcal{F}_{g}$ of the potential $\mathcal{F}$ and find the higher genus versions of the string and divisor equations. 


\section{$3 \quad Q H^{*}(G / B)$ and quantum Toda lattices}

The following proposition has been so far the most efficient tool for computing relations in quantum cohomology algebras. Introduce the following formal vector-function of $q$ with values in the cohomology algebra $H^{*}(X)$ and depending on the formal parameter $\hbar^{-1}$ :

$$
J:=e^{(p \ln q) / \hbar}\left(1+\hbar^{-1} \sum_{d \neq 0} \operatorname{ev}_{*} \frac{q^{d}}{\hbar-c}\right)
$$

where $\mathrm{ev}_{*}$ is the push-forward along the evaluation map ev : $X_{1, d} \rightarrow X$, and $p \ln q=\sum p_{i} \ln q_{i}$. In fact $J$ is determined by the conditions $\left\langle J, \phi_{\beta}\right\rangle=S_{0 \beta}$ where we assume that $\phi_{\alpha}$ with the index $\alpha=0$ is the unity $1 \in H^{*}(X)$. Thus components of the vector-function $J$ form the "1-st row" in the fundamental solution matrix $\left(S_{\alpha \beta}\right)$ of the differential system (3).

Proposition. Suppose that a polynomial differential operator $D\left(\hbar q \frac{\partial}{\partial q}, q, \hbar\right)$ annihilates the vector-function $J$. Then the relation $D(p \circ, q, 0)=0$ holds true in the quantum cohomology algebra $Q H^{*}(X)$.

Proof. Application of the operator $D$ to the fundamental solution matrix $S$ of the system (3) yields $\left(M_{0}+\hbar M_{1}+\ldots+\hbar^{N} M_{N}\right) S$ where $M_{i}$ are some matrix-functions of $q$ and $M_{0}=D(p \circ, q, 0)$. By the hypothesis the 1-st row in the product vanishes and thus the 1-st row in each $M_{i}$ vanishes too since the fundamental solution matrix $S$ is non-degenerate. In particular the entries $\left\langle 1, D(p \circ, q, 0) \phi_{\beta}\right\rangle=\left\langle D(p \circ, q, 0), \phi_{\beta}\right\rangle$ of the 1-st row in $M_{0}$ are all zeroes and thus $D(p \circ, q, 0)=0$.

The proposition indicates that quantum cohomology is a quasi-classical limit of the actual quantum object — the differential system (3). We will illustrate applications of the proposition to computation of $Q H^{*}(X)$ with the example (due to B. Kim (1996)) of the flag manifold $X=G / B$ of a complex semi-simple Lie group $G$ (here $B$ is the Borel subgroup, and the subgroup of unipotent upper-triangular matrices in $S L_{n+1}(\mathbb{C})$ is a good example to have in mind). Roughly speaking,

Kim's theorem identifies $J$ with the ground state of the quantum Toda system corresponding to the Langlands-dual group $G^{\prime}$

(on the level of simple complex Lie algebras the classical series $B_{r}$ and $C_{r}$ are Langlands-dual to each other while all others are self-dual). 
Example: $A_{r}$. The differential operator

$$
H:=\frac{\hbar^{2}}{2} \sum_{i=0}^{r} \frac{\partial^{2}}{\partial t_{i}^{2}}-\sum_{i=1}^{r} e^{t_{i}-t_{i-1}}
$$

is called the hamiltonian operator of the quantum Toda system (corresponding to $\left.S L_{r+1}\right)$. The Hamilton function

$$
\frac{1}{2} \sum_{i=0}^{r} p_{i}^{2}-\sum_{i=1}^{r} e^{t_{i}-t_{i-1}}
$$

on the (complex) phase space with the symplectic structure $\sum d p_{i} \wedge d t_{i}$ defines evolution of the classical Toda system of $r+1$ interacting particles. The Toda system is completely integrable on both classical and quantum levels, and according to Kim's theorem the conservation laws play a key role in the quantum cohomology theory of the manifold

$$
X=\left\{0 \subset \mathbb{C}^{1} \subset \ldots \subset \mathbb{C}^{r} \subset \mathbb{C}^{r+1}\right\}
$$

of complete flags in $\mathbb{C}^{r+1}$.

The cohomology algebra $H^{*}(X)$ is canonically isomorphic to

$$
\mathbb{Q}\left[p_{0}, \ldots, p_{r}\right] /\left(\sigma_{1}(p), \ldots, \sigma_{r+1}(p)\right)
$$

where $p_{i}$ is the 1-st Chern class of the tautological line bundle with the fiber $\mathbb{C}^{i+1} / \mathbb{C}^{i}$ (prove this!).

The flag manifold $X$ has $r$ projections to the partial flag manifolds $X^{(i)}$ defined by omitting the $i$-dimensional space in the flag. The fibers of the projections are isomorphic to $\mathbb{C} P^{1}$ (why?).

Exercise. Prove that any compact holomorphic curve in $X$ of the same degree $\mathbf{1}_{i}$ as the fiber of the projection $X \rightarrow X^{(i)}$ is one of the fibers.

The exercise identifies $X^{(i)}$ with the moduli space $X_{0, \mathbf{1}_{i}}$ of degree $\mathbf{1}_{i}$ rational stable maps to the flag manifold $X$, and also identifies the projection $X \rightarrow X^{(i)}$ with the forgetting map $X_{1, \mathbf{1}_{i}} \rightarrow X_{0,1_{i}}$. The classes $\mathbf{1}_{i}$ form a basis in the lattice $H_{2}(X, \mathbb{Z})$, and we will use the weight $q^{d}$ for contributions of holomorphic curves of degree $\left(d_{1}, \ldots, d_{r}\right)$ with respect to this basis. These notations generalize to arbitrary $X=G / B$ as follows. 
Exercises. (a) Using the Bruhat cell partition of $G / B$ and the spectral sequence of the bundle $B T^{r} \rightarrow B G$ (induced by the embedding of the maximal torus into $G$ ) identify $H^{*}(G / B, \mathbb{C})$ with the algebra of $\mathbb{C}[\mathfrak{g}]^{A d}$ of $A d$-invariants on the Lie algebra of $G$.

(b) Among the subgroups in $G$ containing $B$ (they are called parabolic) there are $r$ sub-minimal parabolic subgroups $P_{1}, \ldots, P_{r}$ (the minimal one is $B$ ) corresponding to simple roots. Put $X^{(i)}=G / P_{i}$ and identify $X^{(i)}$ with $X_{0, \mathbf{1}_{i}}$ and $X \rightarrow X^{(i)}$ - with $\mathrm{ft}: X_{1, \mathbf{1}_{i}} \rightarrow X_{0, \mathbf{1}_{i}}$

(c) Show that the degree of any compact holomorphic curve in $X$ is a non-negative integer combination $\sum d_{i} \mathbf{1}_{i}$.

(d) Using 1-st Chern classes of line bundles identify the lattice of weights of $\mathfrak{g}$ (that is the lattice of characters of the maximal torus in the simply connected model of $G$ ) with $H^{2}(X, \mathbb{Z})$ and show that $\left(\mathbf{1}_{1}, \ldots, \mathbf{1}_{r}\right)$ is a basis of simple roots of the coroot system (which is the root system for $\mathfrak{g}^{\prime}$.

(e) Let $\left(p_{1}, \ldots, p_{r}\right)$ be the dual basis in $H^{2}(X, \mathbb{Z})\left(p_{i}\right.$ are called fundamental weights). Show that $c_{1}\left(T_{X}\right)=2\left(p_{1}+\ldots+p_{r}\right)$ (in other words, $\left.\operatorname{deg} q_{i}=2, i=1, \ldots, r\right)$.

(f) Find the relation of the classes $p_{1}, \ldots, p_{r}$ with what we denoted $p_{0}, \ldots, p_{r}$ in the case of $S L_{r+1}$.

It turns out that the exercise (b) provides enough geometrical information for our purposes about rational curves in the flag manifolds $G / B$.

Lemma 1. Let $Q:=\sum Q_{i j} p_{i} p_{j}=0$ be the quadratic relation in the algebra $H^{*}(X)$ defined by the Killing Ad-invariant quadratic form on $\mathfrak{g}$. Then the relation

$$
Q(p \circ)=\sum Q\left(\mathbf{1}_{k}\right) q_{k}
$$

holds true in the quantum cohomology algebra of $X$.

Proof. For degree reasons $Q(p \circ)$ must be a linear combination $\sum c_{k} q_{k}$. The coefficient $c_{k}$ is then the $\mathrm{GW}$-invariant $\sum Q_{i j}\left\langle p_{i}, p t, p_{j}\right\rangle_{\mathbf{1}_{k}}$ which depends only on the intersection indices of the fiber in $X \rightarrow X^{(k)}$ through the given point $p t$ in $X$ with the divisors $p_{i}$ and $p_{j}$. It equals $\sum Q_{i j} p_{i}\left(\mathbf{1}_{k}\right) p_{j}\left(\mathbf{1}_{k}\right)=Q_{k k}$ since the bases $\left\{p_{i}\right\}$ and $\left\{\mathbf{1}_{i}\right\}$ are dual.

Lemma 2. The differential operator

$$
H:=Q\left(\hbar q \frac{\partial}{\partial q}\right)-\sum Q\left(\mathbf{1}_{k}\right) q_{k}
$$

annihilates $J$.

Proof. Application of $H$ to the fundamental solution matrix $S$ yields

$$
\left[Q(p \circ)-\sum Q\left(\mathbf{1}_{i}\right) q_{i}+\hbar \sum Q_{i j} q_{i} \frac{\partial}{\partial q_{i}}\left(p_{j} \circ\right)\right] S .
$$


The 1-st two terms annihilate each other by Lemma 1 , and the sum of order $\hbar$ has zero 1-st row since the 1-st row entries $\left\langle 1, p_{j} \circ \phi_{\beta}\right\rangle=\left\langle p_{j}, \phi_{\beta}\right\rangle$ in the matrix $p_{j}$ o do not depend on $q$.

Lemma 3. A formal series I of the form

$$
e^{(p \ln q) / \hbar} \sum_{d \geq 0} P_{d} q^{d}
$$

with $P_{d} \in H^{*}\left(X, \mathbb{Q}\left[\hbar^{-1}\right]\right)$ which satisfies the differential equation $H I=0$ is uniquely determined by $P_{0}$.

Proof. The equation $H I=0$ gives rise to the recursion relation

$$
Q(p+\hbar d) P_{d}=\sum_{k} Q\left(\mathbf{1}_{k}\right) P_{d-\mathbf{1}_{k}}
$$

for the coefficients, and $Q(p+\hbar d)$ is invertible in $H^{*}(X)$ for $d \neq 0$ since $p_{i}$ are nilpotent and $Q(d)>0$.

Corollary 1. The $G W$-descendent $J$ for the flag manifold $X=G / B$ is uniquely determined by the differential equation $H J=0$ and the initial condition $P_{0}=1$.

Corollary 2. Let $D\left(\hbar q \frac{\partial}{\partial q}, q, \hbar\right)$ be a polynomial differential operator commuting with $H$ and suppose that the principal symbol $D(p, 0,0)$ vanishes in the algebra $H^{*}(G / B)$. Then $D J=0$ and therefore $D(p \circ, q, 0)=0$ in $Q H^{*}(G / B)$.

Proof: The hypotheses about $D$ guarantee that $I=D J$ satisfies $H I=0$ and has zero initial term $P_{0}$.

Example: $A_{r}$. Consider the characteristic polynomial $\lambda^{r+1}+D_{1} \lambda^{n}+$ $\ldots+D_{r} \lambda+D_{r+1}$ of the matrix

$$
\left[\begin{array}{ccccc}
\frac{\partial}{\partial t_{0}} & e^{t_{1}-t_{0}} & 0 & \ldots & 0 \\
-1 & \frac{\partial}{\partial t_{1}} & e^{t_{2}-t_{1}} & 0 & \ldots \\
0 & -1 & \frac{\partial}{\partial t_{3}} & e^{t_{3}-t_{2}} & \ldots \\
0 & \cdot & \cdot & \cdot & \\
0 & \cdots & 0 & -1 & \frac{\partial}{\partial t_{n}}
\end{array}\right]
$$

Exercise. (a) Should we worry about non-commutative determinants? 
(b) Express $H$ in terms of $D_{1}$ and $D_{2}$.

(c) Check that symbols of the differential operators $D_{1}, \ldots, D_{r+1}$ Poisson-commute.

(d) Prove that $\left[H, D_{i}\right]=0$ for all $i$.

Taking $e^{t_{i}-t_{i-1}}$ on the role of $q_{i}$ and replacing the derivations $\hbar \partial / \partial t_{i}$ in the above matrix by $p_{i}$ we obtain the following

Theorem. ${ }^{3}$ Quantum cohomology algebra of the manifold of complete flags in $\mathbb{C}^{r+1}$ is canonically isomorphic to the algebra

$$
\mathbb{Q}\left[p_{0}, \ldots, p_{r}, q_{1}, \ldots, q_{r}\right] /\left(D_{1}(p, q), \ldots, D_{r+1}(p, q)\right)
$$

of regular functions on the invariant Lagrangian variety of the classical Toda lattice.

For general flag manifolds $G / B$ the differential operators commuting with $H$ come from the theory of quantum Toda lattices on $G^{\prime}$. Consider holomorphic functions $f: G^{\prime} \rightarrow \mathbb{C}$ which transform equivariantly under left translations by the "lower-triangular" unipotent subgroup $N_{+}$and right translations by the "upper-triangular" unipotent subgroup $N_{-}$in accordance with given generic characters $\xi_{ \pm}: N_{ \pm} \rightarrow \mathbb{C}^{\times}$:

$$
f\left(x_{+}^{-1} g x_{-}\right)=\xi_{+}\left(x_{+}^{-1}\right) f(g) \xi_{-}\left(x_{-}\right) .
$$

Restriction of such a function to the maximal complex torus in $G^{\prime}$ (which will be the configuration space of the Toda lattice) determines $f$ on a dense subset in $G^{\prime}$. The commuting differential operators - quantum conservation laws of the Toda lattice - originate of course from the center $Z$ of the universal enveloping algebra $U \mathfrak{g}^{\prime}$. The algebra $U \mathfrak{g}^{\prime}$ identifies with the algebra of, say, left-invariant differential operators on $G^{\prime}$. Its center consists of bi-invariant differential operators on $G^{\prime}$ and thus preserves the sheaf of equivariant functions described above. Thus $Z$ acts on functions on the maximal torus by commuting differential operators.

In fact the bi-invariant laplacian on the group gives rise to the hamiltonian operator $H$ via this construction accompanied by "the $\rho$-shift " conjugation by the multiplication operator $q^{\rho}$ where $\rho$ is the semi-sum of positive roots of the Lie algebra $\mathfrak{g}^{\prime}$. The center $Z$ is known to be isomorphic to the algebra of $A d$-invariant polynomials on $\mathfrak{g}^{\prime}$ through the Harish-Chandra

\footnotetext{
${ }^{3}$ See A. G. \& B. Kim, I. Ciocan-Fontanine, B. Kim (1996).
} 
isomorphism. This gives us $r$ commuting polynomial differential operators $D(p, q, \hbar)$ which are known to have $W$-invariant principal symbols $D(p, 0,0)$ after the conjugation by $q^{\rho} .{ }^{4}$ Thus, combining the above lemmas with known results of representation theory about quantum Toda lattices, B. Kim (1996) proves:

$Q H^{*}(G / B)$ is isomorphic to the algebra of regular functions

$$
\mathbb{Q}\left[p_{1}, \ldots, p_{r}, q_{1}, \ldots, q_{r}\right] /\left(D_{1}(p, q), \ldots, D_{r}(p, q)\right)
$$

where $\left(D_{1}, \ldots, D_{r}\right)$ is the complete set of homogeneous conservation laws of the Toda system with the Hamilton function $Q(p)-\sum Q\left(\mathbf{1}_{k}\right) q_{k}$.

Of course, these conservation laws can be obtained not only as symbols of the commuting differential operators but also form $A d$-invariant polynomials on $\mathfrak{g}^{\prime}$ by suitable symplectic reduction of $T^{*} G^{\prime}$ with respect to the left-right translations by $N_{+} \times N_{-}$.

Exercises. (a) Express the geometrical construction of the commuting differential operators in algebraic terms of the universal enveloping algebra and compute the operator generated by the bi-invariant laplacian. Choose the characters $\xi_{ \pm}$so that after the $\rho$-shift the operator coincides with $H$. (In fact the algebraic language of $U \mathfrak{g}^{\prime}$ is more suitable for observing the necessary polynomiality properties of our differential operators.)

(b) Give enumerative interpretation of the relation $H(p, q)=0$ in $Q H^{*}(X)$.

\section{Singularity theory}

In quantum cohomology theory we have encountered a linear pencil of flat connections

$$
\text { (4) } \nabla_{\hbar}:=\hbar d-\sum A_{i}(t) d t_{i} \wedge
$$

on a trivial vector bundle with the fiber $H$ over some base $B$. Given such a pencil one can ask about asymptotical behavior of horizontal sections as $\hbar \rightarrow$ 0 . It is natural to suspect that the asymptotical behavior should be related to some geometry associated with the operator-valued 1-form $A^{1}$. We will study this geometry under the semi-simplicity assumption that the common eigenvectors of the commuting operators $A_{i}(t)$ form a basis $\left\{v^{\alpha}(t)\right\}, \alpha=1, \ldots, N$ for each $t \in B$. We may also assume (for the sake of our applications) that

\footnotetext{
${ }^{4}$ see B. Kostant (1974) M. Semenov-Tian-Shansky (1987), or B. Kim (1996). By the way, the invariance property of the symbols with respect to the Weyl group $W$ at $q=0$ is a consequence of the theory of Verma modules and their BGG-resolutions.
} 
the operators $A_{i}$ are symmetric with respect to the constant coefficient inner product $\langle\cdot, \cdot\rangle$, and that the eigen-basis is orthonormal. In our actual situation $H$ is the cohomology space of $X$ and contains a distinguished element 1 . The inner product on $H$ is carried over to the algebra $\mathbb{C}[B]\left[A_{1}, \ldots, A_{r}\right]$ as $\langle\phi, \psi\rangle:=\left\langle 1, \phi\left(A_{\text {. }}\right) \psi\left(A_{\text {. }}\right) 1\right\rangle$ and automatically satisfies the Frobenius property.

Proposition (see for instance A. G. \& B. Kim). The eigen-value 1-forms $\sum p_{i}^{\alpha}(t) d t_{i}$ are closed and thus form a Lagrangian variety $L$ in the cotangent bundle $T^{*} B$ with $N$ branches over the base $B$.

Roughly speaking, the proposition means that the subalgebra in $Q H^{*}(X)$ generated by the degree 2 classes can be always treated as the algebra of functions on a Lagrangian variety. The invariant Lagrangian variety in the phase space of the Toda lattice provides a good example.

Exercises. (a) Prove the proposition by differentiating the constant function $w_{\alpha}\left(v^{\alpha}\right)=$ 1 where $w^{\alpha}(t)$ is the corresponding common eigen-covector of operators $A_{i}(t)$.

(b) Give another proof: diagonalize the 1-form, $A^{1}=\Psi D^{1} \Psi^{-1}$, and derive $d D^{1}=0$ from $d A^{1}=0$. Notice that this proof require stronger assumptions than (a).

(c) Show that $L$ is given by the characteristic equation $\operatorname{det}\left(A^{1}-\sum p_{i} d t_{i}\right)=0$ (to be satisfied for all values of the commuting coordinates $d t_{i}$ on the tangent space $T_{t} B$ ) at least if the eigen-value 1-forms $D_{\alpha}^{1}$ are everywhere distinct.

(d) Identify the commutative algebra $\mathbb{C}[B]\left[A_{1}, \ldots, A_{r}\right]$ with the algebra of functions on $L$ (at least under the same hypotheses as in (c)).

(e) Show that the basis of delta-functions of the branches in $L$ diagonalizes the inner product $\langle\phi, \psi\rangle$ in the algebra:

$$
\langle\phi, \psi\rangle(t)=\sum_{p^{\alpha} \in L \cap T_{t}^{*} B} \frac{\phi\left(p^{\alpha}\right) \psi\left(p^{\alpha}\right)}{\Delta\left(p^{\alpha}\right)}
$$

where $\Delta$ is a suitable function on $L$. Show that in the quantum cohomology setting the function $\Delta /(\operatorname{dim} H)$ represents the cohomology class Poincare-dual to a point.

The class in $H^{*}(X \times X)$ Poincare-dual to the diagonal defines an element in $\mathbb{C}\left[L \times_{B} L\right]$. Show that $\Delta$ is the restriction of this element to the diagonal $L \subset L \times_{B} L$. Compare both descriptions of $\Delta$ with the residue formula for Poincare pairing in $Q H^{*}\left(\mathbb{C} P^{n}\right)$ from the exercise in the section 1.

(f) Consider the function $u$ on $L$ defined as a (local) potential $\int \sum p_{i} d t_{i}$ of the eigenvalue 1-forms. Taking into account the grading in quantum cohomology algebras show that the restriction to $L$ of the linear function $c_{1}\left(T_{X}\right)=\sum \mu_{i} p_{i}$ plays the role of such a potential.

Now let us try to find an asymptotical representation of a fundamental solution $S$ to the differential system $\nabla_{\hbar} S=0$ in the form

$$
S=\Psi(t)(1+\hbar \Phi(t)+o(\hbar)) \exp (U / \hbar)
$$


of the product of a formal matrix series in $\hbar$ and the exponential function of the diagonal matrix $U / \hbar$. Equating the terms of order $\hbar^{0}$ in the equation $\nabla_{\hbar} S=0$ we obtain $A^{1} \Psi=\Psi d U$ which means that columns of $\Psi$ must be eigen-vectors of $A^{1}$ and the entries of the diagonal matrix $U$ must be potentials $u_{\alpha}$ of the eigen-value 1 -forms. In the order $\hbar^{1}$ we have $\Psi^{-1} d \Psi=$ $[d U, \Phi]$. Since commutator with a diagonal matrix has zero diagonal entry, this means that in the variation $\Psi(t)$ of an (orthogonal !) eigen-basis of $A^{1}$ inner squares of the eigen-vectors may not vary.

Exercise. Proceed to higher orders in $\hbar$ in order to show that the asymptotical fundamental solution in the form $\Psi\left(1+\hbar \Phi_{1}+\hbar^{2} \Psi_{2}+\ldots\right) \exp (U / \hbar)$ exists.

Reformulating the result of our computation in terms of the algebra of functions on $L$ we conclude that the system $\nabla_{\hbar} \vec{s}=0$ has a basis of solutions with the asymptotical expansion $\vec{s}_{\alpha}=e^{u\left(p^{\alpha}(t)\right) / \hbar}\left(\Delta^{1 / 2}\left(p^{\alpha}\right)(1+o(\hbar))\right.$, and that the corresponding component $\left\langle 1, \vec{s}_{\alpha}\right\rangle$ of the vector-function $J$ assumes the form

$$
J_{\alpha}=\frac{e^{u_{\alpha} / \hbar}}{\sqrt{\Delta_{\alpha}}}(1+o(\hbar))
$$

This form strongly resembles stationary phase asymptotics of oscillating integrals in singularity theory - the subject we have to discuss next.

Let $\pi: Y \rightarrow B$ be a family of complex manifolds $Y_{t}$ and $f: Y \rightarrow \mathbb{C}$ be a holomorphic function. One defines the Lagrangian variety $L \subset T^{*} B$ parametrized by critical points of functions $f_{t}:=f \mid Y_{t}$ as follows. A critical point is a point in $y \in Y$ where the differential $d_{y} f$ is projectable to a covector $p(y)$ on $B$ applied at $t=\pi(y)$. Since $f_{t}$ may have several critical points, we obtain several covectors in $T_{t}^{*} B$. The pull-back of the action 1-form $\sum p_{i} d t_{i}$ to the critical set by the map $y \rightarrow p(y)$ is the differential of the critical value function $y \rightarrow f(y)$, and thus the variety $L \subset T^{*} B$ swept by the covectors $p(y)$ is isotropic and under some mild genericity assumptions - Lagrangian. It is called the Lagrangian variety generated by the family $\left(Y_{t}, f_{t}\right)$. Notice that the algebra of functions on $L$ can be considered as a family of finite-dimensional algebras $H_{t}:=\mathbb{C}\left[Y_{t}\right] /\left(\partial f_{t} / \partial y\right)$ of functions on the critical sets.

Suppose now that $Y_{t}$ are provided with a holomorphic family $\omega_{t}$ of holomorphic volume forms. Then one can define the Hessian $\Delta(y)$ of $f_{t}$ at a critical point $y$ as the determinant of the Hess matrix $\left(\partial^{2} f_{t} / \partial y^{2}\right)$ with respect to a unimodular local coordinate system (which requires that $\omega_{t}(y)=$ 
$\left.d y_{1} \wedge \ldots \wedge d y_{m}, m=\operatorname{dim} Y\right)$. If $f_{t}$ has only non-degenerate critical points one can introduce the residue pairing of functions on $Y_{t}$

$$
\langle\phi, \psi\rangle=\sum_{y \in \operatorname{crit}\left(f_{t}\right)} \frac{\phi(y) \psi(y)}{\Delta(y)}
$$

which makes $H_{t}$ a Frobenius algebra. The residue pairing can be also described by the integral residue formula

$$
\langle\phi, \psi\rangle=\frac{1}{(2 \pi i)^{m}} \oint_{\left\{\left|\partial f_{t} / \partial y_{j}\right|=\epsilon_{j}\right\}} \frac{\phi \psi \omega_{t}}{\frac{\partial f_{t}}{\partial y_{1}} \ldots \frac{\partial f_{t}}{\partial y_{m}}} .
$$

In this form the residue pairing extends to the functions $f_{t}$ with any isolated singularities and is known to remain non-degenerate as a bilinear form on the algebra $H_{t}$.

Consider now the complex oscillating integral of the form

$$
I(t)=\int e^{f_{t} / \hbar} \omega_{t}
$$

over a real $m$-dimensional cycle $\Gamma_{t}$ in $Y_{t}$. It is a function on $B$, and one can study the dependence of $I$ in $t$ by deriving differential equations for it in the following manner.

Exercise. Differentiating the 1-dimensional integral $I=\int e^{\left(y^{3} / 3-t y\right) / \hbar} d y$ derive the equation $\hbar^{2} \ddot{I}=t I$. Compare the symbol $p^{2}=t$ of the equation with the equation of the critical set in the family of phase functions $f_{t}=y^{3} / 3-t y$.

In general the coincidence observed in the exercise is true only asymptotically when $\hbar \rightarrow 0$. Differentiating the integral by $\hbar \partial / \partial t_{i}$ yields an amplitude factor $\phi=\partial f_{t} / \partial t_{i}+o(\hbar)$. At the same time differentiation $\hbar \partial / \partial y_{j}$ along the fibers of the family $Y_{t}$ yields the factor $\partial f_{t} / \partial y_{j}+o(\hbar)$ but does not change the value of the integral. Thus, performing computations modulo $\hbar$ we would conclude that differentiation of $I$ by $\hbar \partial / \partial t_{i}$ is equivalent to multiplication by $\partial f_{t} / \partial t_{i}$ in the algebra $H_{t}$. (Notice that the analogue of this statement in the quantum cohomology theory holds precisely and not only modulo $\hbar$ ).

Furthermore, the stationary phase approximation to the integral $I$ near a critical point $y$ yields

$$
I \sim \hbar^{m / 2} \frac{e^{f_{t}(y) / \hbar}}{\sqrt{\Delta(y)}}(1+o(\hbar))
$$


Exercise. The Gaussian integral $\int_{-\infty}^{\infty} \exp \left(-a y^{2} / \hbar\right) d y$ with positive $a$ and $\hbar$ is proportional to $\hbar^{1 / 2} / a^{1 / 2}$. Expand the integral

$$
\int_{-\infty}^{\infty} e^{\left(f(0)-a y^{2}+b y^{3}+c y^{4}+\ldots\right) / \hbar}\left(1+\alpha y+\beta y^{2}+\ldots\right) d y
$$

into the asymptotical series $\sim \hbar^{1 / 2} e^{f(0) / \hbar} a^{-1 / 2}(1+o(\hbar))$. Show that the integral $\int e^{-a y^{2} / \hbar} \phi(y) d y$ with $\phi$ vanishing identically in a neighborhood of $y=0$ is a flat function of $\hbar$ at $\hbar=0$. Give higher-dimensional generalizations of these statements.

The similarity between asymptotical solutions to the system $\nabla_{\hbar} S=0$ arising from quantum cohomology theory and asymptotics of complex oscillating integrals suggests the following, rather optimistic, conjecture:

Given a compact (almost) Kähler manifold $X$ of complex dimension $m$, one can associate to it a family $\left(Y_{q}, f_{q}, \omega_{q}\right)$ of algebraic $m$-dimensional manifolds, functions and complex volume forms parametrized by the complex torus $H^{2}(X, \mathbb{C}) / H^{2}(X, 2 \pi i \mathbb{Z})$ in such a way that the gravitational descendent $J$ corresponding to $X$ satisfies the same differential equations as the complex oscillating integral $I$, that is

$$
\left\langle J, \phi_{\alpha}\right\rangle=\int_{\Gamma_{\alpha}} e^{f_{q} / \hbar} \omega_{q}
$$

for suitable bases of classes $\phi_{\alpha}$ in $H^{*}(X)$ and of cycles $\Gamma_{\alpha}$ in $Y_{q}$.

It is interesting to look at this formulation in the degenerate case when the manifold $X$ is algebraic and has zero 1-st Chern class $c_{1}\left(T_{X}\right)$ (such $X$ are called Calabi-Yau manifolds in a broad sense, and abelian manifolds, elliptic curves, K3-surfaces and their higher-dimensional generalizations provide a pool of examples). It is expected that the manifolds $Y_{t}$ in this case are also compact and therefore the functions $f_{t}$ are constant. Yet $Y_{t}$ should carry a non-vanishing holomorphic $m$-form $\omega_{t}$ and thus must have zero 1-st Chern class as well. The oscillating integrals degenerate to the periods $\int \omega_{t}$ of the volume forms which are known to distinguish non-equivalent complex structures. On the other hand, since $Y_{t}$ are compact algebraic manifolds, one can define their GW-invariants, quantum cohomology algebras, etc. Then variations of complex structures detected by periods of complex volume forms on $X$ are expected to represent GW-invariants of $Y$. This symmetric picture of mirror correspondence between Calabi-Yau manifolds is the classical (and somewhat oversimplified) version of the mirror conjecture. In fact the relation between symplectic (resp. complex) geometry of $X$ and complex (resp. 
symplectic) geometry of $Y$ is expected to be much more profound than the equality between periods $I$ of holomorphic forms and the solution $J$ of our differential system.

With the same reservations, we can interpret the above conjecture as a proposal to generalize the mirror conjecture beyond the class of CalabiYau manifolds. As it follows from our asymptotical analysis of the system $\nabla_{\hbar}=0$, one should admit non-compact manifolds $Y_{q}$ provided with nonconstant function $f_{q}$ on the role of mirror partners and be prepared to sacrifice the symmetry of the mirror correspondence.

Exercise. Let $X$ be a non-singular degree 5 hypersurface in $\mathbb{C} P^{4}$. Show that it is a Calabi-Yau manifold, that its quantum cohomology algebra is nilpotent, the Lagrangian variety $L$ is a multiple zero section in $T^{*} B$ and the differential equation for $J$ does not really depend on $\hbar$. Generalize these observations to arbitrary CY manifolds. Compare these results with properties of complex "oscillating" integrals with constant phase functions.

The actual motivation of the generalized mirror conjecture comes from supporting examples based on toric geometry. Here is the simplest one.

Example: the mirror of $\mathbb{C} P^{1}$. The integral

$$
I=\int_{\Gamma \subset\left\{y_{1} y_{2}=q\right\}} e^{\left(y_{1}+y_{2}\right) / \hbar} \frac{d y_{1} \wedge d y_{2}}{d q}
$$

satisfies the Bessel differential equation

$$
\left(\hbar q \frac{\partial}{\partial q}\right)^{2} I=q I
$$

and therefore $\left(Y_{q}, f_{q}, \omega_{q}\right)$ where $Y_{q}$ is given by the equation $y_{1} y_{2}=q$ in $Y=\mathbb{C}^{2}$, the function $f_{q}$ is the restriction to $Y_{q}$ of $f=y_{1}+y_{2}$, and $\omega_{q}$ is the relative "volume" form $\left(d y_{1} \wedge d y_{2}\right) / d\left(y_{1} y_{2}\right)$ on $Y_{q}$ can be taken on the role of the mirror partner of $X=\mathbb{C} P^{1}$. In greater detail, let $\hbar>0, q \neq 0$. The function $f_{q}$ in the coordinate $y_{1} \neq 0$ on $Y_{q}$ reads $f_{q}=y_{1}+q / y_{1}$ and has two critical points $y_{1}= \pm q^{1 / 2}$ with the critical values $\pm 2 q^{1 / 2}$. On the line of values of $f_{q}$ pick two paths starting from the critical values and going to infinity toward the direction $\operatorname{Re} f_{q} \rightarrow-\infty$. Each such a path has two preimages in $Y_{q}$ which glue up to a non-compact cycle when oriented oppositely. The integral $I$ over each of the two cycles (denote them $\Gamma_{ \pm}$) converges. As a function of $q$ it satisfies the Bessel differential equation. Indeed, in logarithmic coordinates 
$T_{i}=\ln y_{i}, t=\ln q$ the integral takes on the form

$$
\int_{\tilde{\Gamma}_{ \pm} \subset\left\{T_{1}+T_{2}=t\right\}} e^{\left(e^{T_{1}}+e^{T_{2}}\right) / \hbar} \frac{d T_{1} \wedge d T_{2}}{d\left(T_{1}+T_{2}\right)},
$$

and the double differentiation $\hbar^{2} \partial^{2} / \partial T_{1} \partial T_{2}$ yields the amplitude factor $e^{T_{1}+T_{2}}$ $=q$. The variety $L$ generated by the family $f_{q}$ is described by the relation $p^{2}=q$ in $Q H^{*}\left(\mathbb{C} P^{1}\right)$. The potential $u=\int p d \ln q$ of the action 1 -form coincides with the the critical value function $\pm 2 q^{1 / 2}=2 p$. Since the Hessian $\Delta(p)$ of $f_{q}=e^{T_{1}}+q e^{-T_{1}}$ at the critical points equals $2 p$, the residue pairing defines the Frobenius structure on $\mathbb{C}[L]$ identical to the Poincare pairing $(2 \pi i)^{-1} \oint d p \phi \psi /\left(p^{2}-q\right)$.

Exercises. (a) Using holomorphic version of the Morse lemma show that all critical points of the real part of a holomorphic Morse function in $m$ variables have the same Morse index $m$. Deduce that under some transversality assumptions about a holomorphic function $f: Y \rightarrow \mathbb{C}$ at infinity the rank of the relative homology group $H_{m}(Y, \operatorname{Re} f \rightarrow$ $-\infty)$ equals the total multiplicity of critical points. Generalize to higher dimensions the construction of the cycles $\Gamma_{ \pm}$from the above example. Show that in the example the cycles $\Gamma_{ \pm}$form a basis in the group $H_{1}\left(Y_{q}, \operatorname{Re} f_{q} \rightarrow-\infty\right)$. Find the place for the compact cycle $\left|y_{1}\right|=1$ in this group.

(b) Prove that $\left(Y_{q}, f_{q}, \omega_{q}\right)$ with

$$
Y_{q}: y_{1} \ldots y_{n+1}=q, f_{q}=\left(y_{1}+\ldots+y_{n+1}\right) \mid Y_{q}, \omega_{q}=\frac{d y_{1} \wedge \ldots \wedge d y_{n+1}}{d\left(y_{1} \ldots y_{n+1}\right)}
$$

is the mirror partner of $X=\mathbb{C} P^{n}$ in the same sense as in the case $n=1$ studied in the example.

\section{Toda lattices and the mirror conjecture}

The example of the mirror partner for $\mathbb{C} P^{1}$ can be generalized to the manifold $X$ of complete flags in $\mathbb{C}^{r+1}$ as follows.

Consider the following "2-dimensional Toda lattice" with $(r+1)(r+2) / 2$ 
vertices and $r(r+1)$ edges:

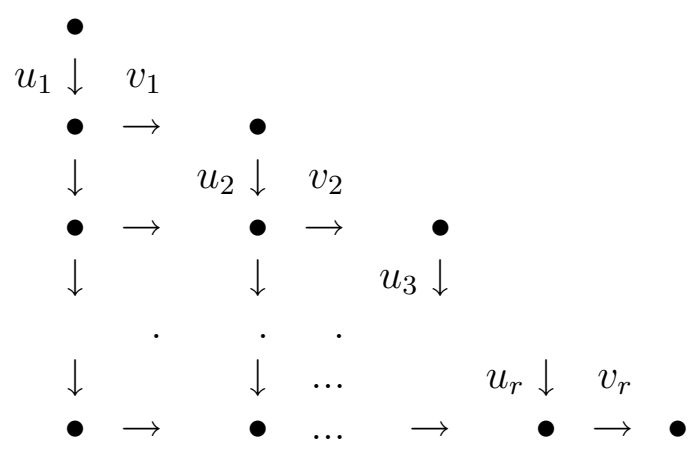

For each edge $\epsilon$ of the lattice we introduce a complex $y_{\epsilon}$. For each $1 \times 1$-square

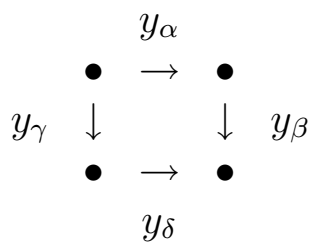

we impose the "commutativity" relation $y_{\alpha} y_{\beta}=y_{\gamma} y_{\delta}$. These relations determine the variety $Y$ of complex dimension $r+r(r+1) / 2$ in the space with coordinates $y_{\epsilon}$. Using the notation $u_{i}, v_{i}$ (as shown on the diagram) for the variables $y_{\epsilon}$ corresponding to the edges next to the diagonal, we fiber $Y$ over the space $B$ with coordinates $q_{1}, \ldots, q_{r}$ :

$$
q_{1}=u_{1} v_{1}, \ldots, q_{r}=u_{r} v_{r}
$$

For $q_{1} \ldots q_{r} \neq 0$ all the relations together mean that all $y_{\epsilon}$ are non-zero and that their logarithms satisfy the Kirchhoff law: the voltage drops $\ln y_{\epsilon}$ accumulate to 0 over a closed contour, and $\ln q_{i}$ determine the voltage drop between neighbor diagonal vertices. Thus one can express $y_{\epsilon}$ via the vertex "potentials" - variable $T_{\nu}$ corresponding to the vertices $\nu$ of the lattice: $y_{\epsilon}=\exp \left(T_{\nu_{+}}-T_{\nu_{-}}\right)$where $\nu_{+}$and $\nu_{-}$are respectively the head and the tail of the edge $\epsilon$. In particular, the variables $T_{\nu}$ corresponding to all under-diagonal vertices form a coordinate system on the covering of the complex torus $Y_{q}$. We put

$$
\omega_{q}=\wedge_{\nu} d T_{\nu}
$$


and define $f_{q}$ as the restriction to $Y_{q}$ of the function

$$
f=\sum_{\text {all edges } \epsilon} y_{\epsilon}=\sum_{\epsilon} e^{T_{\nu_{+}(\epsilon)}-T_{\nu_{-}(\epsilon)}} .
$$

Theorem. ${ }^{5}$ Complex oscillating integrals

$$
I=\int_{\Gamma \subset Y_{q}} e^{f_{q} / \hbar} \omega_{q}
$$

satisfy the differential equations $D_{1} I=\ldots=D_{r+1} I=0$ where $D_{i}(\hbar q \partial / \partial q, q)$ are the quantum conservation laws of the quantum Toda lattice associated with the group $S L_{n+1}$.

Corollary. The family $\left(Y_{q}, f_{q}\right)$ generates in $T^{*} B$ the invariant Lagrangian variety $D_{1}(p, q)=\ldots=D_{r+1}(p, q)=0$ of the classical Toda lattice.

Exercise. Check that in the case $r=1$ the theorem agrees with the example of the mirror partner for $\mathbb{C} P^{1}$.

Even the corollary is not quite obvious. We will prove it by induction on the number of diagonals in our 2-dimensional lattice. Let us recall that that the operator $D:=\lambda^{r+1}+D_{1} \lambda^{r}+\ldots+D_{r+1}$ is the characteristic polynomial of the matrix introduced in the section 3 . Denote $t_{0}, t_{1}, \ldots t_{r}$ the vertex variables $T_{\nu}$ corresponding to the diagonal vertices. Since $\partial f / \partial t_{i}=v_{i}-u_{i+1}$ and $q_{i}=u_{i} v_{i}$, we need to prove that the characteristic polynomial of the following matrix equals $\lambda^{n+1}$ at critical points of $f_{q}$ :

$$
A_{r+1}=\left[\begin{array}{ccccc}
-u_{1} & u_{1} v_{1} & 0 & \ldots & \\
-1 & v_{1}-u_{2} & u_{2} v_{2} & 0 & \ldots \\
0 & -1 & v_{2}-u_{3} & u_{3} v_{3} & \ldots \\
& \cdot & \cdot & \cdot & \\
& \cdots & 0 & -1 & v_{r}
\end{array}\right]
$$

The matrix $A_{r+1}$ factors into the product $U V$ of the following square matri-

\footnotetext{
${ }^{5}$ See A. Givental, Stationary phase integrals, quantum Toda lattices, flag manifolds and the mirror conjecture.
} 
ces:

$$
U=\left[\begin{array}{cccccc}
u_{1} & 0 & \ldots & & & \\
1 & u_{2} & 0 & \ldots & & \\
0 & 1 & u_{3} & 0 & \ldots & \\
& . & . & . & & \\
& \ldots & 0 & 1 & u_{r} & 0 \\
& & & 0 & 1 & 0
\end{array}\right], V=\left[\begin{array}{cccccc}
-1 & v_{1} & 0 & \ldots & & \\
0 & -1 & v_{2} & 0 & \ldots & \\
0 & 0 & -1 & v_{3} & 0 & \ldots \\
& . & . & . & & \\
& & \ldots & 0 & -1 & v_{r} \\
& & \ldots & & 0 & -1
\end{array}\right]
$$

Since $V$ is invertible, the matrix $U V$ is similar to $B=V U$. We find:

$$
B=\left[\begin{array}{cccccc}
v_{1}-u_{1} & v_{1} u_{2} & 0 & \ldots & & 0 \\
-1 & v_{2}-u_{2} & v_{2} u_{3} & 0 & \ldots & 0 \\
0 & -1 & v_{3}-u_{3} & v_{3} u_{4} & \cdots & 0 \\
& \cdot & \cdot & \cdot & & 0 \\
& \cdots & 0 & -1 & v_{r}-u_{r} & 0 \\
& & \cdots & 0 & -1 & 0
\end{array}\right]
$$

We claim that the characteristic polynomial of $B$ equals $\lambda^{n+1}$ by the induction hypothesis. Indeed, using "commutativity" of the $1 \times 1$-squares next to the diagonal of the lattice and the criticality conditions $0=\partial f / \partial T_{\nu}=$ $\sum_{\epsilon: \nu_{ \pm}(\epsilon)=\nu} \pm y_{\epsilon}$ at the vertices $\nu$ next to the diagonal we can identify the upper left $r \times r$ corner of the matrix $B$ with the matrix $A_{r}$ corresponding to the 2-dimensional lattice with the main diagonal cut off. By the induction hypothesis $\operatorname{det}\left(\lambda+A_{r}\right)=\lambda^{r}$ under the conditions $\partial f / \partial T_{\nu}=0$ at all other under-diagonal vertices $\nu$.

The prove of the theorem can be obtained as a non-commutative version of the above inductive argument. Application of the operator $D$ to the function $\exp (f / \hbar)$ yields the amplitude factor $\operatorname{det}\left(\lambda+A_{r+1}\right)$ which is, as we already know, equivalent to $\lambda^{r+1}$ modulo the ideal generated by the partial derivatives $\partial f / \partial T_{\nu}$ along directions tangent to the fibers $Y_{q}$. Derivatives in these directions annihilate the integral $I$, but the equivalence modulo the ideal is not sufficient: we need to earn the same equivalence modulo exact forms by honest consecutive differentiation. This plan can be completed without complications. However, the actual meaning of the integral $I$ in harmonic analysis on $S L_{r+1}$ and in the theory of quantum Toda lattices remains unclear. Generalizations of this mirror construction to the flag manifolds $G / B$ of other semi-simple groups are also unknown. 
Exercise. Thinking of 2-dimensional lattices of a block-triangular shape, guess mirror partners of the manifolds of partial flags in $\mathbb{C}^{r+1}$, starting with $\mathbb{C} P^{r}$ and grassmannians. Check that your answer for $\mathbb{C} P^{r}$ gives rise to the same mirror partner as in the section 4 .

Remark. The "right" answer to the exercise remains only a guess: although quantum cohomology algebras of partial flag manifolds have been described and their conjectural mirrors - found, the differential equations for $I$ and $J$ have not been identified so far.

\section{References}

[1] V. I. Arnold. Sur un propriété topologique des applications canoniques de la mechanique classique. C. R. Acad. Sci. Paris 261 (1965), 3719 - 3722.

[2] V. Batyrev, I. Ciocan-Fontanine, B. Kim \& D. van Straten. Conifold transitions. Preprint, Mittag-Leffler Inst., 1997.

[3] K. Behrend \& B. Fantechi. The intrinsic normal cone. Invent. Math. (1997).

[4] K. Behrend \& Yu. Manin. Stacks of stable maps and Gromov-Witten invariants. Duke Math. J. 85 (1996), 1 - 60.

[5] I. Ciocan-Fontanine. On quantum cohomology rings of partial flag varieties. Institut Mittag-Leffler Report No. 12, 1996/1997.

[6] C. C. Conley \& E. Zehnder. The Birkhoff-Lewis fixed point theorem and a conjecture of V. I. Arnold. Invent. Math. 73 (1983), 33 - 49.

[7] R. Dijkgraaf, E. Verlinde \& H. Verlinde. Notes on topological string theory and $2 D$ quantum gravity. In "String Theory and Quantum Gravity", M. Green et al. (Eds.) World-Scientific, Singapore, 1991, 91 - 156.

[8] B. Dubrovin. The geometry of $2 D$ topological field theories. In "Integrable Systems and Quantum Groups", Lect. Notes in Math. 1620, Springer-Verlag, Berlin, 1996, $120-348$.

[9] T. Eguchi, K. Hori \& C.-S. Xiong. Gravitational quantum cohomology. Preprint, 1996.

[10] A. Floer. Morse theory for Lagrangian intersections. J. Diff. Geom. 28 (1988), 513 -547 .

[11] A. Floer. Symplectic fixed points and holomorphic spheres. Commun. Math. Phys. 120 (1989), $575-611$.

[12] B. Fortune \& A. Weinstein. A symplectic fixed point theorem for complex projective spaces. Bul. Amer. Math. Soc. 12 (1985), 128 - 130.

[13] K. Fukaya \& K. Ono. Arnold conjecture and Gromov-Witten invariants. Preprint, 1996, 155 pp. 
[14] A. Givental. Homological geometry and mirror symmetry. In "Proceedings of the International Congress of Mathematicians, 1994, Zürich ", Birkhäuser, Basel, 1995, $472-480$.

[15] A. Givental. Stationary phase integrals, quantum Toda lattices, flag manifolds and the mirror conjecture. In "Topics in Singularity Theory", A. Khovansky, A. Varchenko, V. Vassiliev (Eds.), Adv. in Math. Sci., AMS, Providence, RI, 1997, 103 -116 .

[16] A. Givental \& B. Kim. Quantum cohomology of flag manifolds and Toda lattices. Commun. Math. Phys. 168 (1995), $609-641$.

[17] M. Gromov. Pseudo-holomorphic curves in symplectic manifolds. Invent. Math. 82 (1985), $307-347$.

[18] B. Kim. On equivariant quantum cohomology. IMRN (1996), No. 17, $841-851$.

[19] B. Kim. Quantum cohomology of flag manifolds $G / B$ and quantum Toda lattices. Preprint, 1996.

[20] B. Kim. Quantum Lefschetz principle. Preprint, 1997.

[21] M. Kontsevich. Enumeration of rational curves via toric actions. In "The moduli space of curves", R. Dijkgraaf, C. Faber, G. van der Geer (Eds.), Progr. in Math. 129, Birkhäuser, Boston, 1995, 335 - 368 .

[22] B. Kostant. On Whittaker vectors and representation theory. Invent. Math. 48 (1978), $101-184$.

[23] J. Li \& G. Tian. Virtual Moduli cycles and Gromov-Witten invariants in general symplectic manifolds. Preprint, alg-geom/9608032.

[24] Y. Ruan. Virtual neighborhoods and pseudo-holomorphic curves. Preprint, 1996, 83 pp.

[25] M. Semenov-Tian-Shansky. Quantization of Toda lattices. In "Dynamical systems 7", V. Arnold, S. Novikov (Eds.), Encyclopaedia of Math. Sci. 16, Springer-Verlag.

[26] E. Witten. Supersymmetry and Morse theory. J. Diff. Geom. 117 (1982), 353 - 386.

[27] E. Witten. Two-dimensional gravity and intersection theory on moduli space. Surveys in Diff. Geom. 1 (1991), $243-310$. 